\title{
Experimental Machine Vision System for Training Students in Virtual Instrumentation Techniques
}

\author{
http://dx.doi.org/10.3991/ijoe.v7iS2.1706 \\ R. Holonec, R. Copindean, F. Dragan, and A.O. Buzura \\ Technical University of Cluj-Napoca, Romania
}

\begin{abstract}
The aim of this paper is to present the main techniques in designing and building of a complex machine vision system in order to train electrical engineering students in using virtual instrumentation. The proposed test bench realizes an automatic adjustment of some electrical circuit parameters on a belt conveyer. The students can learn how to combine mechanics, electronics, electrical engineering, image acquisition and processing in order to solve the proposed application. After the system implementation the students are asked to present in which way they can modify or extend the system for industrial environment regarding the automatic adjustment of electric parameters or the calibration of different type of sensors (of distance, of proximity, etc) without the intervention of the human factor in the process.
\end{abstract}

Index Terms-machine vision, stepper motors, automatic adjustment, virtual instrumentation

\section{INTRODUCTION}

The students from the "Instrumentation and Data Acquisition" specialty from our Electrical Engineering Faculty have the opportunity to study virtual instrumentation in our laboratory. Here they can experiment different applications using various types of hardware and software, mainly National Instruments devices and LabVIEW. The proposed machine vision system is useful for our students in learning how to design it in order to meet the next requirements: on a conveyer belt, boards with identical electrical circuits are moved in front of a camera. The system must automatically adjust the current into each circuit.

The first thing the students have do is to identify the components of machine vision system and their main requirements. The word system means an assembly of elements (mechanical, optical, electric, electronic) depending on one another, forming an organized whole and making a practical activity function according to the targeted aim. From a chronological point of view, there have been simple systems conducted by a human operator, programmed systems that behave in a certain way (more advanced than the first ones) and finally systems with sensorial properties, with an ability of planning, recognition of shapes, navigation and learning (with possibilities of advanced information processing), i.e. systems with artificial intelligence. These have the possibility to alter their behavior according to the changes in the internal and external environment.

Intelligent systems interact with their environment by means of some inputs (information, energy, material, me- chanical actions exerted by the system over the environment).

Among several types of intelligent systems structures, the most adequate architecture is the network type presented in [1].

The three main functions of such a system are pointed out in Fig. 1: function of perception, of knowledge and that of execution.

The perception function has the role to collect, to store, to process and distribute information about the existing machine state and also about the environment in which it operates. In order to accomplish this function, sensors, transducers and data acquisition systems are used.

The knowledge function consists in planning and initiating the system actions taking into account the information provided by the perception systems. By using this function a certain evolution of the system is chosen, this way offering long term advantages: minimum material expenses, resources, energy, self-maintenance, etc. The computer vision, machine vision and image processing are included in the category of such intelligent systems.

A Machine Vision system is an automatic system, able to make decisions based on the analysis of different geometrical or topographical characteristics of the analyzed "scene". Generally, the "scene" is a simple or complex physical product, usually an object (a bottle, for instance) or a mechanical assembly, but it can also be an image of different nature. The Machine Vision is appropriate for the automatic quality control, industrial automatic inspections, 2D and 3D automatic measurements, optical characters recognition (OCR), optical character verification (OCV), automatic measurements and selections. Uncertain sources are represented by the appearance of unexpected and unforeseen events and by the incomplete and insufficient information needed to decide what it has to be done.

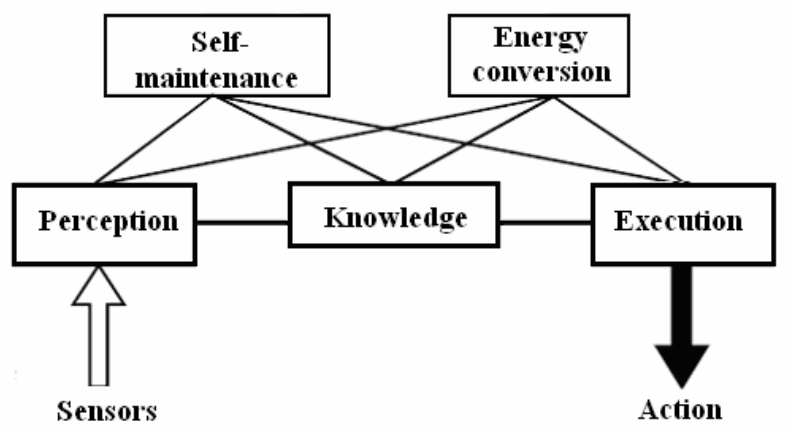

Figure 1. Network architecture for an intelligent system 
Image Processing is a domain of artificial intelligence that takes care of the way of presentation, restoration, classification, recognition and analysis of images by means of a computing system. The source of images may be an acquisition device (video camera, scanner, radar) but it can also be a mathematic equation, an assembly of statistic data, etc. There are two main reasons for which one appeals to processing images [2]:

-The quality improvement of an image with the purpose of a better view for a human operator. This may mean: noise reduction (due for instance to the acquisition device), pointing out some areas of interest by altering the luminosity, contrast, focusing on the edges, etc.

-The information extraction from an image, information that may represent the input for an automatic system of recognition and classification. These pieces of information may be: different distances and relations among the existing objects in the image, statistical moments, geometric parameters (area, perimeter, circularity) etc.

The execution process consists of initiating, developing and concluding the actions of the system based on the instructions received from the knowledge and perception subsystems. The main elements in the execution subsystem structure are the actuators [3], [4]. These elements develop moments and forces and accomplish the mechatronic system movements. The category of actuators specific to the applications in mechatronics has to be noticed: dc servomotors, stepper motors, synchronous or asynchronous machines, pneumatic, hydraulic, electrohydraulic elements of execution etc.

An important element of machine vision systems is the lighting of the objects [5], [6]. It is well known that the quality and suitability of lighting are critical aspects for creating a robust and timed vision inspection. The application of some techniques requires a specific light and geometry, or relative placement of the camera, object, and light. In general, effective solutions can be chosen between back lighting, diffuse lighting (full bright field), bright field (partial bright field or directional) and dark field [3]. These techniques help the developers to better direct their time, effort, and resources in the vision system design, testing, and implementation.

\section{EXPERIMENTAL SYSTEM FOR AUTOMATIC ADJUSTMENT OF ELECTRICAL PARAMETERS ON A CONVEYER BELT}

The aim of the application is to teach the students to design and build, on a reduced scale, a machine vision system. For that, an experimental test bench has to be built (Fig. 2) in order to measure and automatically adjust the potentiometer value in the circuit illustrated in Fig. 2.a.

The test bench is, in fact, a computer numerical control (CNC) machine with 4 axes. As a consequence, four actuators (stepper motors-mpp) must be used as follows: the first three actuators for positioning the adjustment device (Fig. 2b) in $\mathrm{x}, \mathrm{y}, \mathrm{z}$ directions and the forth actuator for the adjustment device itself (axis $z$ '). The role of the adjustment device is to execute the rotation movement needed to drive the potentiometer.

The actuators and their control electronics represent the execution subsystem in our case. An image of the assembly is presented in Fig 3.
The main characteristic of machine vision system is given by the presence of a video camera that supervises the process of automatic adjustment. In the present application, the video camera together with the image acquisition board forms the perception subsystem.

Last but not least it is the software subsystem that must be implemented entirely in LabVIEW from National Instruments. This software offers the possibility of simple integration of specific acquisition, processing and displaying techniques.

\section{A. The Execution Subsystem}

In Fig. 3 a general view of the system is illustrated. Four stepper motors (mpp) can be used and they act as follows:

- Mpp1-acts on the conveyer belt (axis X). By means of conveyer belt the circuit board with the potentiometer is transported in a proper place where it is adjusted;

- Mpp2-moves the adjustment device to the left and right of the conveyer belt (axis Y);

- Mpp3-moves down and up the adjustment device (axis Z);

- Mpp4-moves the adjustment device itself (axle Z'). The adjustment device is formed by the stepper motor mpp4, the reducer and the screwdriver. Four pins are used to supply the circuit board and also for the current measurement. They enter in contact with the board.

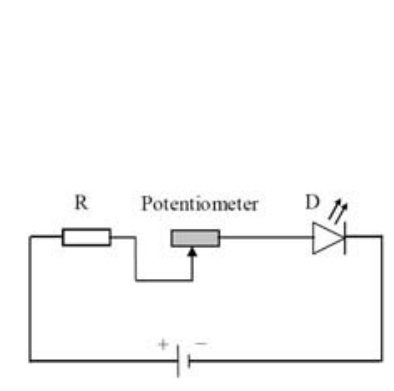

a)

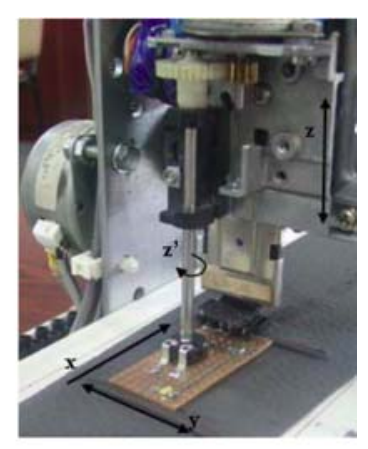

b)
Figure 2. a) Electric circuit b) Adjustment device

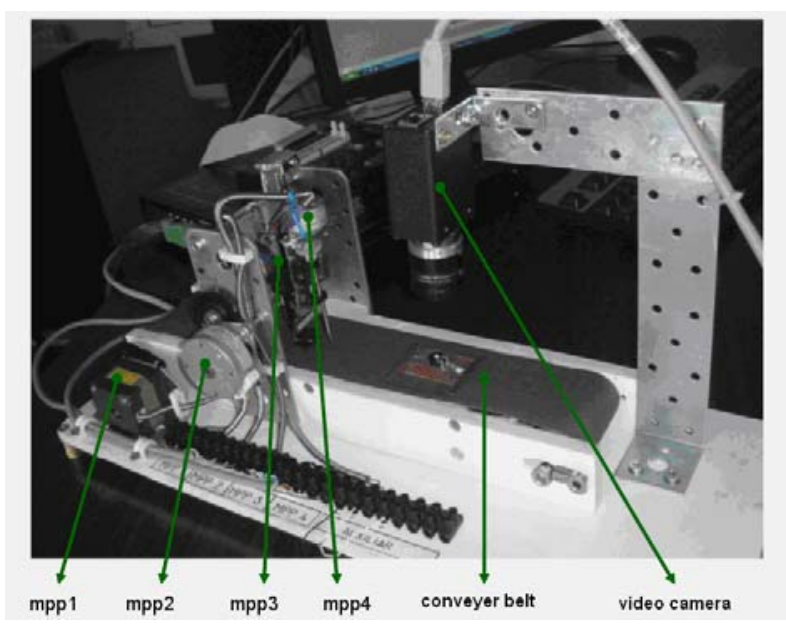

Figure 3. Assemble image on the built system 
The link between the actuators and the driven elements is made by means of reducers with reduction ratios presented in Table I.

The actuators hardware control is also included in the execution subsystem. For this purpose, a module formed by the movement controller 7344 and the power amplifier MID 7604 (from National instruments) has to be used.

\section{B. Perception Subsystem}

In the implementation of the perception subsystem the students shall use a Basler A601f video camera with the following characteristics: 1/2-inch Monochrome CMOS, 656x491 Pixel Array, 60 Frames/Sec Fixed, External Trigger, FireWire (IEEE1694) interface.

The camera is mounted above the belt using a metallic support (Fig. 3) and is triggered the moment the potentiometer reaches its centre coordinates. A single image acquisition is taken and then useful information is extracted to adjust the potentiometer at the needed value.

\section{Knowledge Function}

We remind that the knowledge function consists of planning and initiating the actions of the system taking into account the information ensured by the perception subsystem.

LabVIEW software is used by combining image acquisition and processing techniques with movement control. First, the students open the Measurement And Automation Explorer (MAX) for initiating, configuring, testing and monitoring the controllers/ actuators (stepper motors), then they use Motion Assistant software for actuators control, and then they test the image acquisition and digital processing steps in Vision Assistant. The main program has to be integrated in a single LabVIEW complex virtual instrument.

The movement control refers to the ability to have an accurate control of the position, speed, acceleration, deceleration and the torque needed for positioning the rotating or linear elements. In the case of an automatic process, the control has a major importance, influencing directly the precision and speed of the respective process. The interaction between the hardware and the software for the movement is shown in Fig. 4.

The first subprogram of the movement control must contain the initialization of the controllers and actuators. By using MAX, the students can choose the proper acceleration, deceleration, motion type and the number of steps. The following stage is to do a draft program in Motion Assistant, with parameters from MAX. Then, the draft program must be imported in LabVIEW according to Fig. 5. The same program must be used for all actuators.

The image acquisition and digital processing must be done using the facilities of LabVIEW NI Vision software. A subprogram has to be created to acquire and process the image in order to find the centre coordinates $\mathrm{X}, \mathrm{Y}$ and the angle $Z^{\prime}$ of the adjustment screw (Fig. 6). A pattern matching algorithm must be used.

Besides the two subprograms already mentioned there is another one that deals with feeding the circuit board with a $5 \mathrm{~V}$ voltage and with the measurement of the voltage drop on the fixed value resistor $\mathrm{R}$ from the circuit. This is possible due to the pins that contact the circuit board.
TABLE I.

STEPPER MOTORS CHARACTERISTICS

\begin{tabular}{|c|l|l|l|l|}
\hline & \multicolumn{1}{|c|}{ mpp1 } & \multicolumn{1}{|c|}{ mpp2 } & \multicolumn{1}{c|}{ mpp3 } & \multicolumn{1}{c|}{ mpp4 } \\
\hline Name mpp & $\begin{array}{l}\text { MatsuShita } \\
\text { KP39NM2 }\end{array}$ & $\begin{array}{l}\text { OKI } \\
\text { QR8001 }\end{array}$ & Unknown & $\begin{array}{l}\text { TEC SP- } \\
\text { 25AE24HB }\end{array}$ \\
\hline Type mpp & bipolar & bipolar & Bipolar & bipolar \\
\hline $\begin{array}{c}\text { Feeding } \\
\text { voltage }\end{array}$ & $24 \mathrm{~V}$ & $24 \mathrm{~V}$ & $24 \mathrm{~V}$ & $24 \mathrm{~V}$ \\
\hline $\begin{array}{c}\text { Cur- } \\
\text { rentphase }\end{array}$ & $0.2 \mathrm{~A} /$ phase & $0.2 \mathrm{~A} /$ phase & $\begin{array}{l}0.2 \\
\mathrm{~A} / \mathrm{ph} \text { ase }\end{array}$ & $\begin{array}{l}0.2 \\
\mathrm{~A} / \text { phase }\end{array}$ \\
\hline $\begin{array}{c}\text { Number of } \\
\text { steps }\end{array}$ & 200 & 48 & 48 & 24 \\
\hline $\begin{array}{c}\text { Number of } \\
\text { micro-steps }\end{array}$ & 25000 & 6000 & 6000 & 6000 \\
\hline $\begin{array}{c}\text { Ratio of } \\
\text { transmission }\end{array}$ & 3 & 4.3 & 1 & 2.5 \\
\hline Increment & $\begin{array}{l}0.15-0.0012 \\
\mathrm{~mm}\end{array}$ & $0.02-0.00016$ \\
$\mathrm{~mm}$ & $0.26-$ & $0.002 \mathrm{~mm}$ & $\begin{array}{l}6-0.024 \\
\text { degrees }\end{array}$ \\
\hline
\end{tabular}

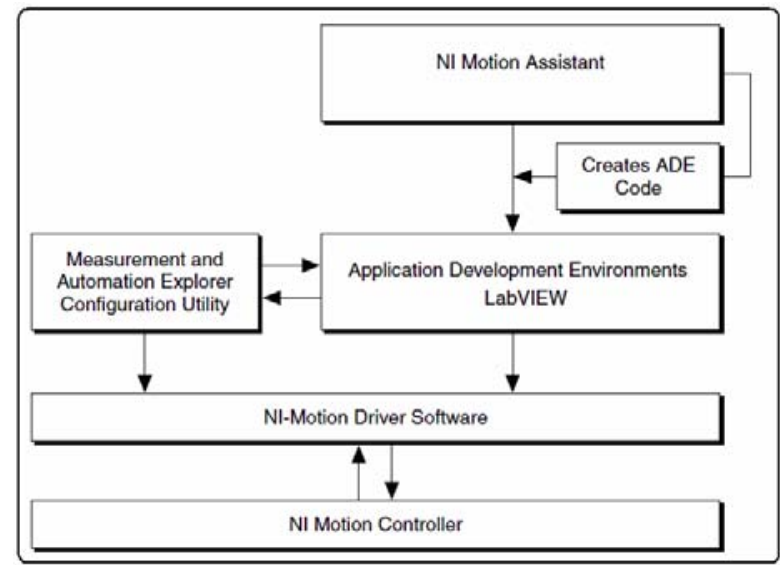

Figure 4. The Motion Control Hardware and Software Interactions

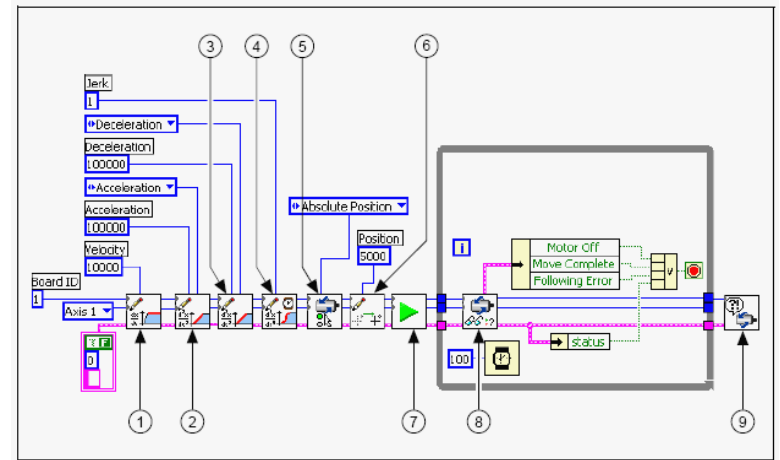

Figure 5. The subprogram dedicated to the motors movement control

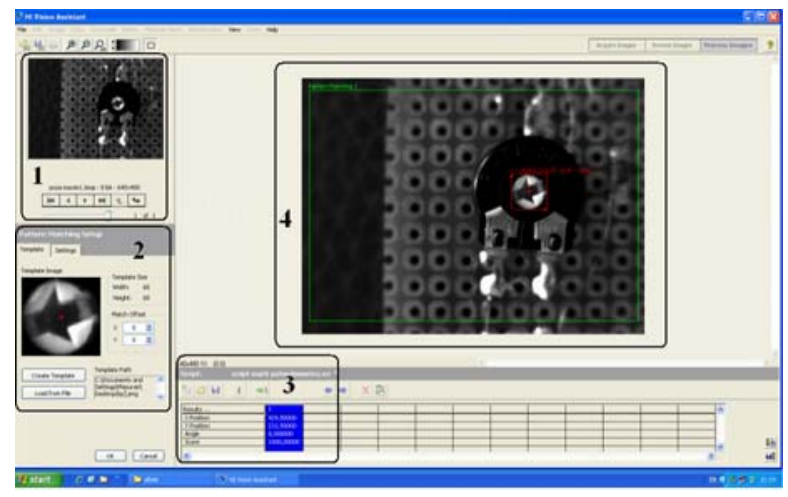

Figure 6. Trimmer Potentiometer Image processing 
The frontal panel of the control instrument has to look like in fig. 7. There are 4 areas of interest:

- Acquired image - the window in which we see images captured by the camera;

- Frame indicators (led) - indicate the frame within which the program is.

- Information regarding the actuators as well as the score with which the template is found;

- Current and Voltage controls - They should be used to control the current in the circuit and the circuit voltage supply

The program must be written to satisfy the next adjustment requirements:

- When the button "Run" is pushed, the band starts moving. The circuit board reaches under the camera;

- The image acquisition and processing must be done. The coordinates $(\mathrm{X}, \mathrm{Y})$ and the angle of the potentiometer adjustment screw are found;

- The positioning of the circuit board under the adjustment device must be done (Mpp1 will do a preset number of steps). The number of steps for mpp2 must be calculated taking into account the difference between the coordinate $\mathrm{X}$ of the potentiometer and the fixed coordination of the adjustment device. The difference expressed in pixels will be then transformed in number of steps and applied to mpp2. The same principle is to be applied for mpp4;

- The down movement of the adjustment device (movement on the axis Z-mpp3) must be done. The number of steps must be calculated;

- The adjustment device must position the potentiometer to a maximum value and then the voltage must be acquisitioned. The current must be calculated by dividing the measured voltage with the resistance value of the fixed resistor;

- Once the potentiometer is adjusted, the device must be lifted by mpp 3 and positioned according to the reference point;

- The adjustment device must be placed at the reference point by mpp 2 and mpp 4 .

\section{EXPERIMENT}

We remind that the main purpose is to teach students to design and build a test bench having a machine vision structure. No certain precision, speed or pre-defined times in which the regulation process takes place were imposed. After building the system, the students must test and analyze it. They must observe different errors that can appear, they must correct them and they are asked to find optimal solutions.

Concerning the programming architecture, the students are asked to modify the application by using a state machine method. The state machine will constantly be executing one case or another, even if it is only a default or timeout case. They must also find different front panel design in order to have a friendly user interface.

The students are finally asked to propose other applications using the same hardware components. They must make a report on the realized machine vision system specifying the possibilities to extend it into the industrial field.

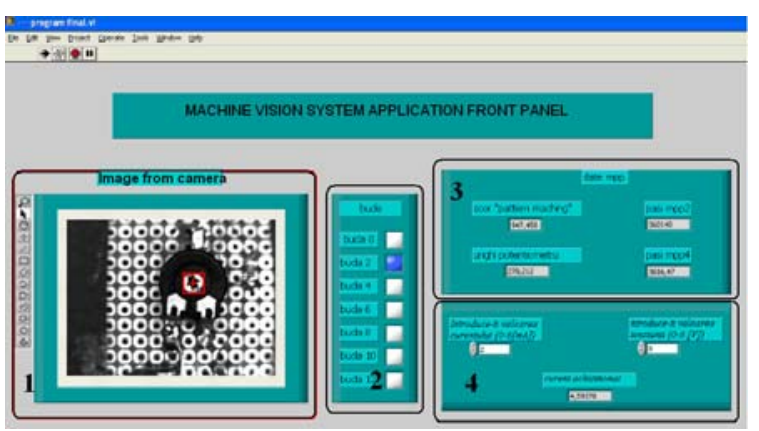

Figure 7. Application Front Panel

\section{CONCLUSION}

Modern machine vision systems typically contain faster and more powerful PC platforms easy-to-use integrated software applications, making machine vision systems more powerful, easier to program, and less expensive to use.

Machine vision can be used in a wide variety of manufacturing operations for repetitive inspection tasks in which accuracy and reliability are important. To take full advantage of machine vision systems and to easily integrate them into the manufacturing lines, it is best to take some time to learn the basics about a vision system, how it is implemented, and the importance of proper planning. Knowing the goals and understanding the parameters upfront will yield improved vision-system design [7].

Because the machine vision applications are so diverse, specific components can vary from system to system. In our virtual instrumentation application the students are put in the situation to use optics, lighting, sensors, actuators, video cameras, a PC platform and inspection software. The proposed application can be integrated in systems where the user wants to adjust automatically the electric parameters or to calibrate different sensors, without human intervention in the process.

\section{REFERENCES}

[1] Th Borangiu, "Intelligent Image Processing in Robotics and Manufacturing", Romanian Academy Publishing House, Bucharest, 2004

[2] R.C. Gonzalez, R.E. Woods, "Digital Image Processing", Prentice Hall, Inc., 2nd Edition, London, 2002, pp. 1-33.

[3] M. Daryl "A Practical Guide to Machine Vision Lighting", Advanced illumination oct 2007

[4] V. Mătieş, M. Mătieş, R.Bălan, "Conceptual Design in Mechatronics“ National Symposium Prasic, Brasov, pp. 49-54, 2002

[5] S. Yi, R.M Haralick, L.G Shapiro, "Automatic Sensor and Light Source Positioning for Machine Vision". Proceedings on 10th Int. Conference on Pattern Recognition, pp. 55-59 vol.1, 1990

[6] U.S. Khan, J. Iqbal, M.A. Khan "Automatic Inspection System using Machine Vision"; Proc Applied Imagery and Pattern Recognition Workshop, pp.210-217, 2005

[7] Christopher Poling, "Designing a machine-vision system " SPIE Newsroom, oemagazine, 30 May 2002.

\section{AUTHORS}

R. Holonec, R. Copindean, F. Dragan, and A.O. Buzura are with Technical University of Cluj-Napoca, Romania.

This article is an extended version of a paper presented at the International Conference on Remote Engineering \& Virtual Instrumentation (REV2011), held at Transylvania University, Brasov, Romania, June 28 July 1, 2001. Received 16 June 2011. Published as resubmitted by the authors 28 September 2011. 